\title{
Las MiPymes como factor determinante del emprendimiento: un análisis bibliométrico para el periodo 2000- 2020
}

\section{SMEs as a determinant factor of entrepreneurship: a bibliometric analysis from 2000 to 2020}

Yolanda Mariana García-Carranza

Universidad Rey Juan Carlos, España

ym.garcia.2016@alumnos.urjc.es

D https://orcid.org/0000-0002-0537-1367

Recepción: 21/07/2021 | Aceptación: 15/10/2021 | Publicación: 30/10/2021

Cómo citar (APA, séptima edición):

García-Carranza, Y.M. (2021). Las Mipymes como factor determinante del emprendimiento: un análisis bibliométrico para el periodo 2000-2020. INNOVA Research Journal, 6(3.1), 79-93.

https://doi.org/10.33890/innova.v6.n3.1.2021.1828

\section{Resumen}

Las micro pequeña y mediana empresa (en adelante MiPymes) han cobrado interés en el campo académico y científico en los últimos 20 años (Contreras, et al., 2016) siendo consideradas como fuerza motora en la generación de empleo y vía de escape para las personas en situación de desempleo y mercados laborales ineficientes. Las MiPymes representan el 99\% de los negocios en Ecuador, según datos de INEC 2017, la mayor concentración se encuentra en el sector servicio y comercio. La presente investigación tiene como objetivo identificar y visualizar la evolución y estructura intelectual de las MiPymes. Se analizan 298 documentos descargados de la plataforma Web of Science. La metodología utilizada fue de tipo descriptiva utilizando técnica cuantitativa como lo es el software SciMAT que permite analizar al campo estructural de nuestra temática de estudio. En el análisis de resultados se detecta que el año 2019 corresponde al de mayor publicación donde destaca la temática Born Global (internacionalización de las pymes desde sus inicios). La principal conclusión determina que los estudios referentes a las pymes están relacionados con cinco temáticas que son: conocimiento, instituciones, internacionalización, tecnología y emprendedorismo, siendo formadas mayoritariamente por núcleos familiares y su principal estrategia de expansión y crecimiento es la exportación de sus productos por la satura del mercado nacional.

Palabras claves: Mipymes; emprendimiento; bibliometría; SciMAT. 


\begin{abstract}
Micro-small and medium-sized enterprises (hereinafter MiPymes) have gained interest in the academic and scientific field in the last 20 years (Contreras, et al., 2016) being considered as a driving force in job creation and a way out for unemployed people and inefficient labor markets. MiPymes represent 99\% of businesses in Ecuador, according to INEC 2017 data, the largest concentration is in the service and commerce sector. The aim of this research is to identify and visualize the evolution and intellectual structure of MSMEs. It analyzes 298 documents downloaded from the Web of Science platform. The methodology used was descriptive, using quantitative techniques such as SciMAT software, which allows us to analyze the structural field of our study. The analysis of results shows that the year 2009 corresponds to the year of greatest publication where the topic Born Global (internationalization of SMEs since their inception) stands out. The main conclusion is that the studies concerning SMEs are related to five themes: knowledge, institutions, internationalization, technology and entrepreneurship, being formed mostly by families and their main strategy of expansion and growth is the export of their products due to the saturation of the national market.
\end{abstract}

Keywords: Mipymes; entrepreneurship; bibliometrics; SciMAT.

\title{
Introducción
}

Las pequeñas y medianas empresas, en adelante (PYMES), desarrollan un papel importante en la economía de los países con la comercialización de los bienes finales que satisfacen las necesidades de la población generando empleo y subempleo a un amplio sector de la Población Económicamente Activa (PEA) (EKOS, 2019).

En los países pertenecientes a la Organización para la Cooperación y Desarrollo Económico, las pymes representan más del 50\% del PIB con ciertas variaciones dependiendo del sector de actividad y del país, son aquellas que pertenecen al sector servicios que aportan como mínimo al 60\% del PIB como lo indica la Organización Internacional de Trabajo (en adelante O.I.T) en su informe anual 2019. Su mayor aporte consiste en ofrecer empleo a personas con difícil acceso al mercado laboral como jóvenes en su primera experiencia laboral, adultos con experiencia senior, personas con capacidades especiales y personas no cualificadas.

Harvey y Wendel (2006); Guapatín (2003); Gómez et al. (2009) citado por (García et al, 2015) señalan que para las PYMES de Latinoamérica el acceso a las fuentes de financiamiento es un problema común y tiende a ser una característica de los países en desarrollo. Se indica además que, a través de los tiempos, estas empresas han tenido un papel preponderante en el desarrollo de las naciones, a pesar de los acelerados cambios tecnológicos, la globalización y las desventajas que enfrentan con respecto a las grandes compañías.

La brecha existente entre las grandes empresas y las MiPymes surge por la inversión realizada en innovación, investigación y desarrollo por parte de las grandes compañías que conlleva a una baja productividad y lento crecimiento de las MiPymes mayoritariamente en los países emergentes. 
En el estudio realizado por la O.I.T (2019) denominado: “El poder de lo pequeño, hay que activar el potencial de las pymes", enlista una serie de perspectivas desde el punto de vista del empleador y empleado en un contexto general de las condiciones adversas de dirigir una pyme en entornos dinámicos y negativos (Tabla 1).

\section{Tabla 1}

Perspectivas del empleador y del empleado de las MiPymes

\begin{tabular}{ll}
\hline Perspectiva del Empleador & Perspectiva del empleado \\
\hline Entornos normativos complejos \\
$\begin{array}{l}\text { Difícil acceso a la financiación } \\
\text { Déficit de competencias laborales }\end{array}$ & $\begin{array}{l}\text { Salarios más bajos } \\
\text { Ausencia de la Seguridad Social } \\
\text { Niveles Insuficientes de seguridad y salud para el } \\
\text { trabajador }\end{array}$ \\
$\begin{array}{l}\text { Dificultad de acceso a la } \\
\text { Relaciones laborales más frágiles }\end{array}$ \\
\hline
\end{tabular}

Fuente: O.I.T., 2019

La tabla 1 indica las perspectivas del empleador y del empleado de una MiPymes en donde destacan dos características relevantes como son que las MiPymes trabajan en entornos dinámicos y complejos y que sus salarios retribuyen por debajo de lo estipulado por los gobiernos.

En el ámbito local, Ecuador se conocen como MiPymes a las micro, pequeñas y medianas empresas que, por su orden jurídico, volumen de ventas y número de trabajadores reúnen las siguientes características (Instituto Nacional de Estadísticas, INEN, 2018):

a) Microempresas: 1 a 9 trabajadores, ventas o ingresos brutos anuales menores a $\$ 100.000$ USD.

b) Pequeña empresa: 10 a 49 trabajadores, ventas o ingresos brutos anuales entre $\$ 100.001$ a $\$ 1^{\prime} 000.000$ USD

c) Mediana empresa: 50 a 199 trabajadores, ventas o ingresos brutos anuales entre $\$ 1$ '000.001 a $\$ 5^{\prime} 000.000$ USD.

Según últimos datos obtenidos en el portal web de la Superintendencia de Compañías del Ecuador (2018), el país tiene un total de 68.520 empresas, de las cuales el 53\% corresponden a la región de la Costa, $45 \%$ corresponden a la Sierra, $2 \%$ al Oriente y $0.4 \%$ a Galápagos. Guayaquil es la segunda ciudad más importante del Ecuador, pero la primera referente actividad comercial en el país existen 8.502 empresas que representan el 33\%, del total, de las cuales el 28\% (43.637) empresas corresponden al sector de Comercio, código G del CIIU (Clasificación Industrial Internacional Uniforme de las actividades económicas, versión 4) y de las empresas comerciales, un 45\% (24.637) son PYMES. 
El comercio es la principal actividad a la que se dedican las pequeñas y medianas empresas del país, ya que constituyen alrededor de un 30\% a la parte comercial. De estos mismos datos el $27 \%$ están formada por pequeñas empresas y el $37 \%$ por las medianas empresas. (Revista Ekos, 2017).

Las PYMES comerciales de Guayaquil, se financian a través de: a) crédito de proveedores $(33 \%)$; b) aportes de socios $(28 \%)$; c) otras cuentas por pagar no relacionadas o préstamos de amigos y terceras personas $(14 \%)$; d) cuentas por pagar relacionados o préstamos de familiares (13\%); e) obligaciones con instituciones financieras (10\%); y, f) anticipos de Clientes (3\%). Así lo especifica la Superintendencia de Compañías del Ecuador (2017).

Las obligaciones con instituciones financieras ocupan un quinto lugar y este tipo de empresas desconocen información referente a fuentes y formas de financiamiento, por tal motivo, no obtienen créditos en el mercado financiero.

Ante lo anteriormente expuesto, el objetivo principal de este trabajo de investigación es conocer la evolución conceptual de las MiPymes como factores determinantes del emprendimiento en el campo científico, académico y empresarial. Para esto, se han descargado artículos que tratan sobre las MiPymes y su relación con el emprendimiento de la plataforma Web of Science (WOS) desde el año 2000 al 2020. Para este análisis se utilizará el software de mapa de la ciencia SciMAT con el propósito de estudiar y evaluar el rendimiento de la producción científica de la temática de estudio, así como visualizar los temas más estudiados en la comunidad académica a partir del análisis de co-ocurrencia de las keywords añadidas en la búsqueda de los documentos.

En la revisión de literatura existente no se evidencia ningún artículo bibliométrico que visualice la evolución conceptual de las MiPymes. Por tanto, la presente investigación contribuye en el campo científico como fuente de información futura detectando posibles líneas de investigación de las lagunas identificadas en la evolución conceptual.

La principal conclusión determina que los estudios relacionados a las Mipymes se enfocan en cinco temas básicos y central, destacando el interés en los últimos cinco años a todo lo relacionado a Born Globals.

Este artículo se divide en tres partes, la primera corresponde a la metodología utilizada en esta investigación, la segunda sección detalla los resultados encontrados, la última sección presenta las conclusiones que se basan en los resultados de investigación y las contribuciones que se generan.

\section{Metodología}

Los estudios bibliométricos como apoyo de la ciencia permiten conocer de manera cuantitativa la producción literaria de una tema o disciplina especifico (Camps, 2007). Para realizar el presente análisis bibliométrico se utilizó la plataforma de búsqueda de información científica 
Web of Science (en adelante W.O.S). Se escogió esta plataforma por ser una de las más utilizadas en el campo académicos para trabajos de investigación por contener publicaciones importantes en las áreas de ciencias, ciencias sociales, artes y humanidades.

La búsqueda de información se realizó en abril de 2021 utilizando los términos: "Small and Medium Entreprise" OR "SMES" AND "Factors entrepreneurship". Se consideraron los siguientes términos de inclusión y exclusión de la búsqueda: a) Todos los periodos excepto 2021, b) tipo de publicación: artículos, c) áreas temáticas: business, economic and management.

Para poder analizar la evolución en el campo investigativo y académico de la temática se utilizó el software Scimat, diseñado para la visualización de mapas científicos. El mapeo científico intentar mostrar la estructura conceptual en un campo de la ciencia determinado (Borner, Chen y Boyack, 2003). Con el software Scimat se puede elaborar y visualizar la estructura conceptual de un campo de investigación (Cobo et al, 2012) dentro de un marco temporal o longitudinal basado en cuatro fases (figura 1):

\section{Figura 1}

Fases de análisis de Scimat
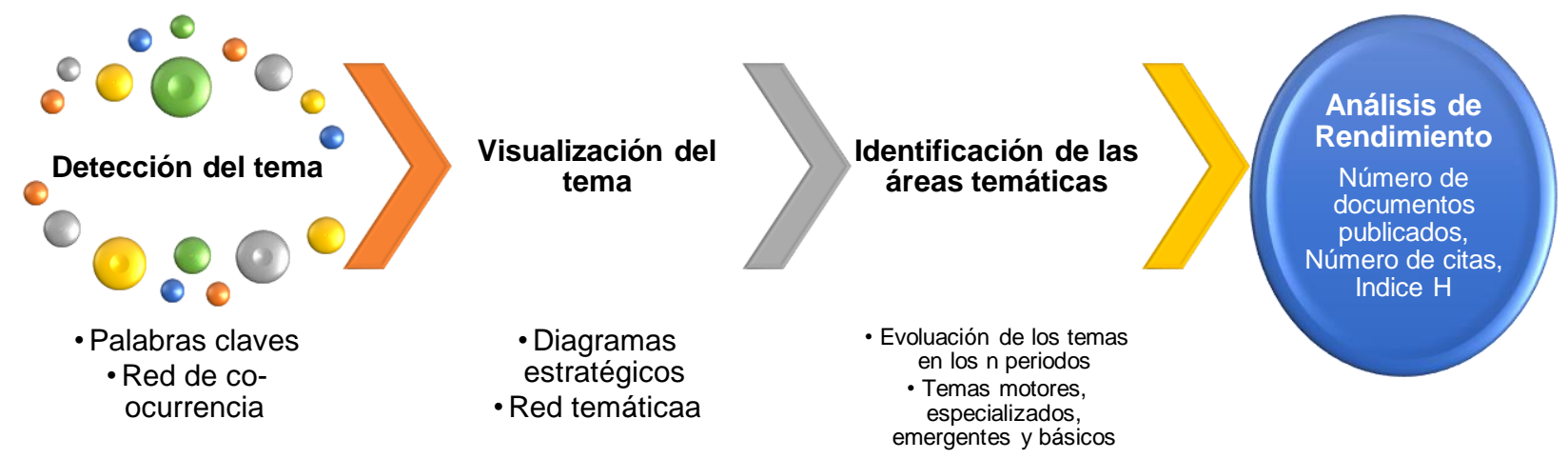

El software Scimat permite realizar estudios basados en análisis bibliométricos por coocurrencia de palabras claves, cocitaciones de revistas, autores y bibliográfica. En este trabajo se utiliza la coocurrencia de las palabras claves que se encuentran en los documentos de estudio. Además, el scimat permite hacer una normalización de los datos antes de sus análisis, debido a la presencia de errores como: duplicaciones de nombres de los autores, palabras claves con el mismo contexto y falta de datos en los años de los artículos de referencia por encontrarse en acceso anticipado lo que se tuvo que añadir manualmente. El arco cronológico se dividió en cuatro periodos: 2000-2004, 2005-2009, 2010-2015, 2016-2020. 


\section{Resultados}

La búsqueda avanzada en la W.O.S. arrojó 298 documentos, de los cuales el $90 \%$ corresponde a investigaciones realizadas en el área de business economic. Para el análisis de los clústeres del software Scimat se eligió solo el nodo donde aparecía SME'S para analizar los temas desarrollados en ese periodo y su relación con las MiPymes.

\section{Figura 2}

Publicaciones por año de la temática

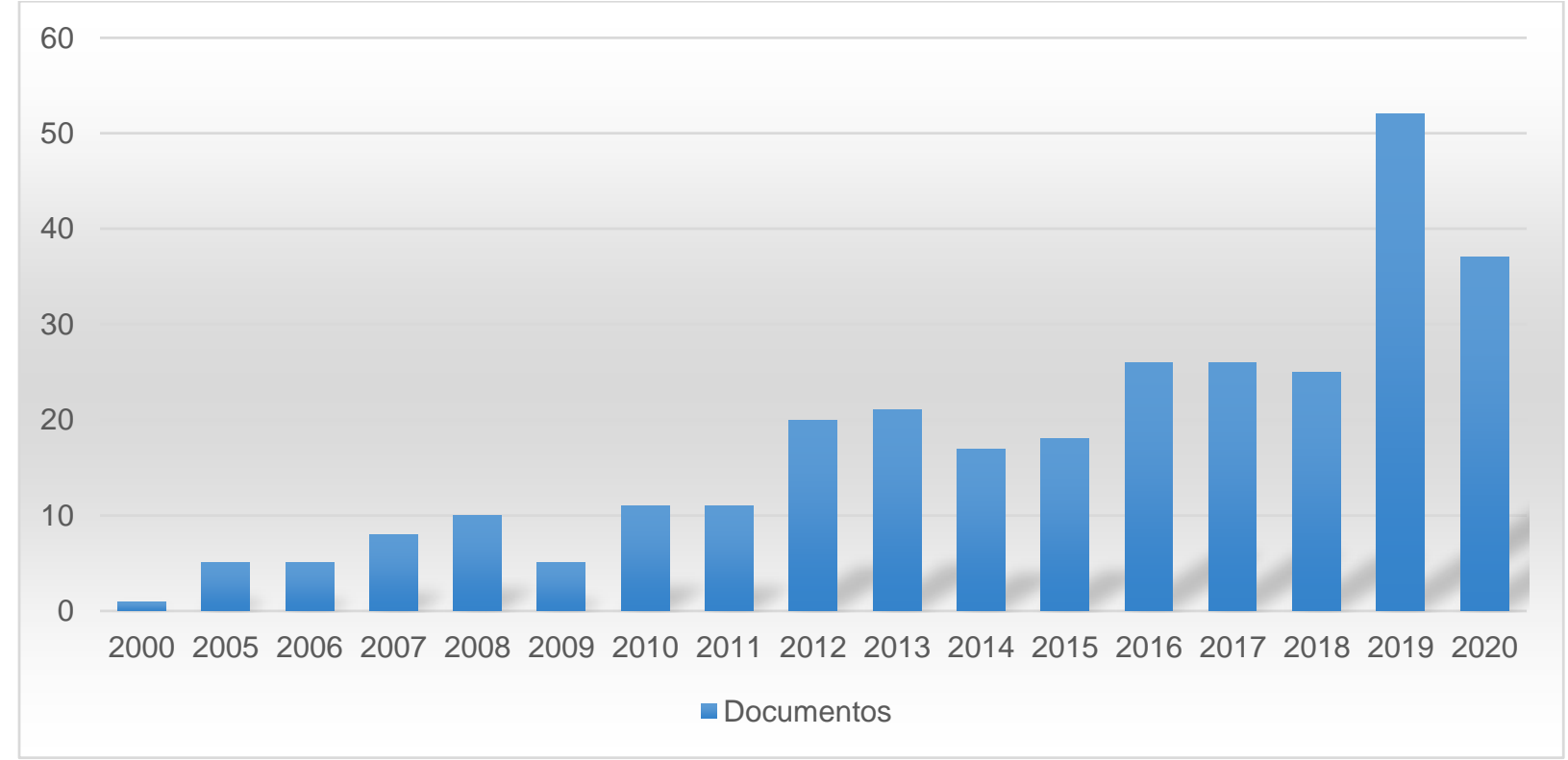

De los datos descargados de la W.O.S. se evidencia (figura 2) que a pesar de que el primer artículo que centra su investigación de las Mypimes y el emprendimiento, no es a partir del año 2005 que genera mayor interés. Aunque la mayor cantidad de artículos publicados es el 2019 donde los temas principales surgen en la innovación, digitalización y la internacionalización de las Mipymes. 


\section{Figura 3}

\section{Autores con más publicaciones}

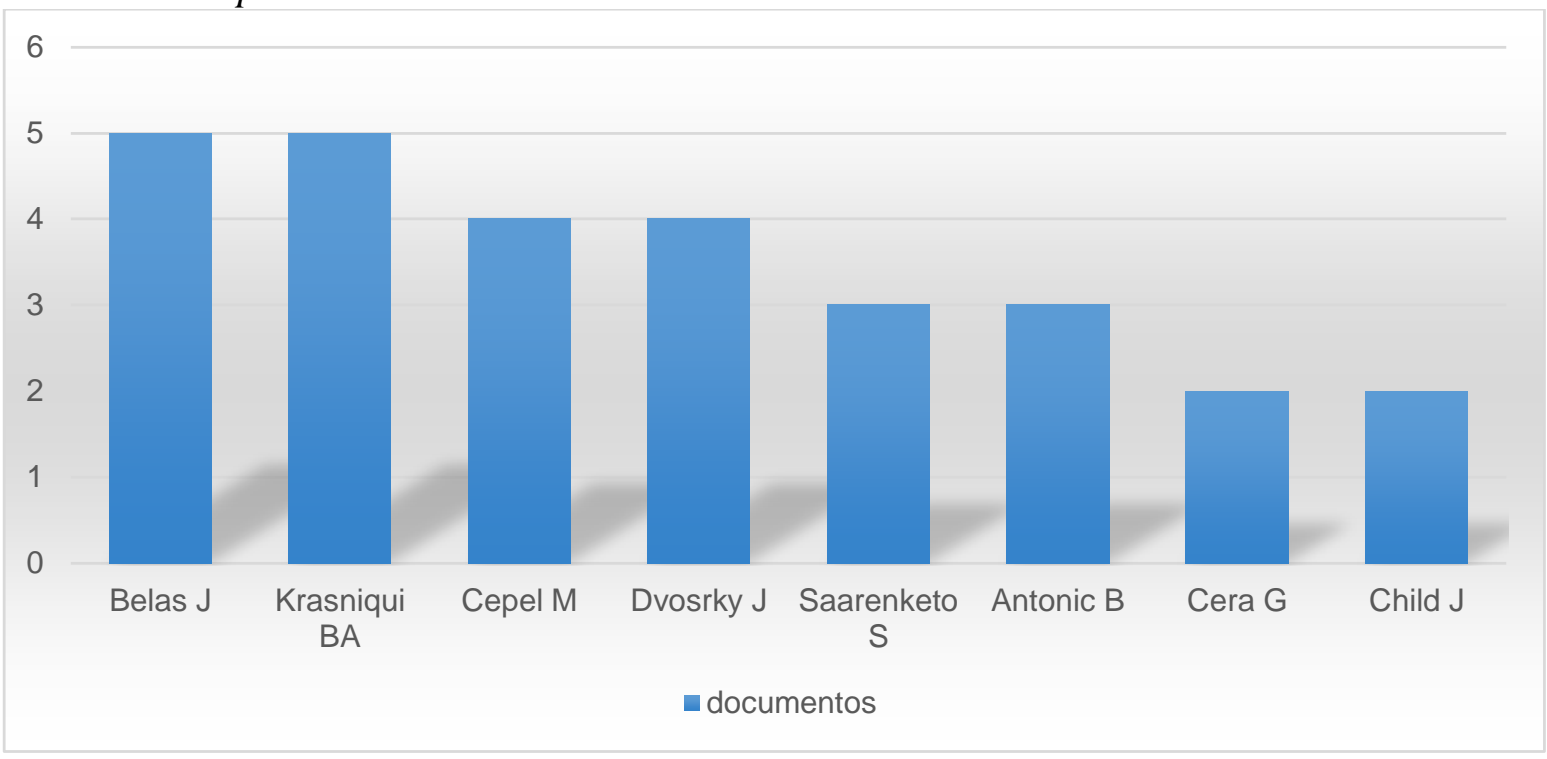

De los 298 documentos se evidencia 636 autores en la figura 3 muestra los principales autores con el mayor número de artículos publicados que son James Belas y Besnik Krasniqi que centran sus investigaciones en el crecimiento económico y las mypimes en economías en transición.

\section{Figura 4}

\section{Países con mayor número de publicaciones}

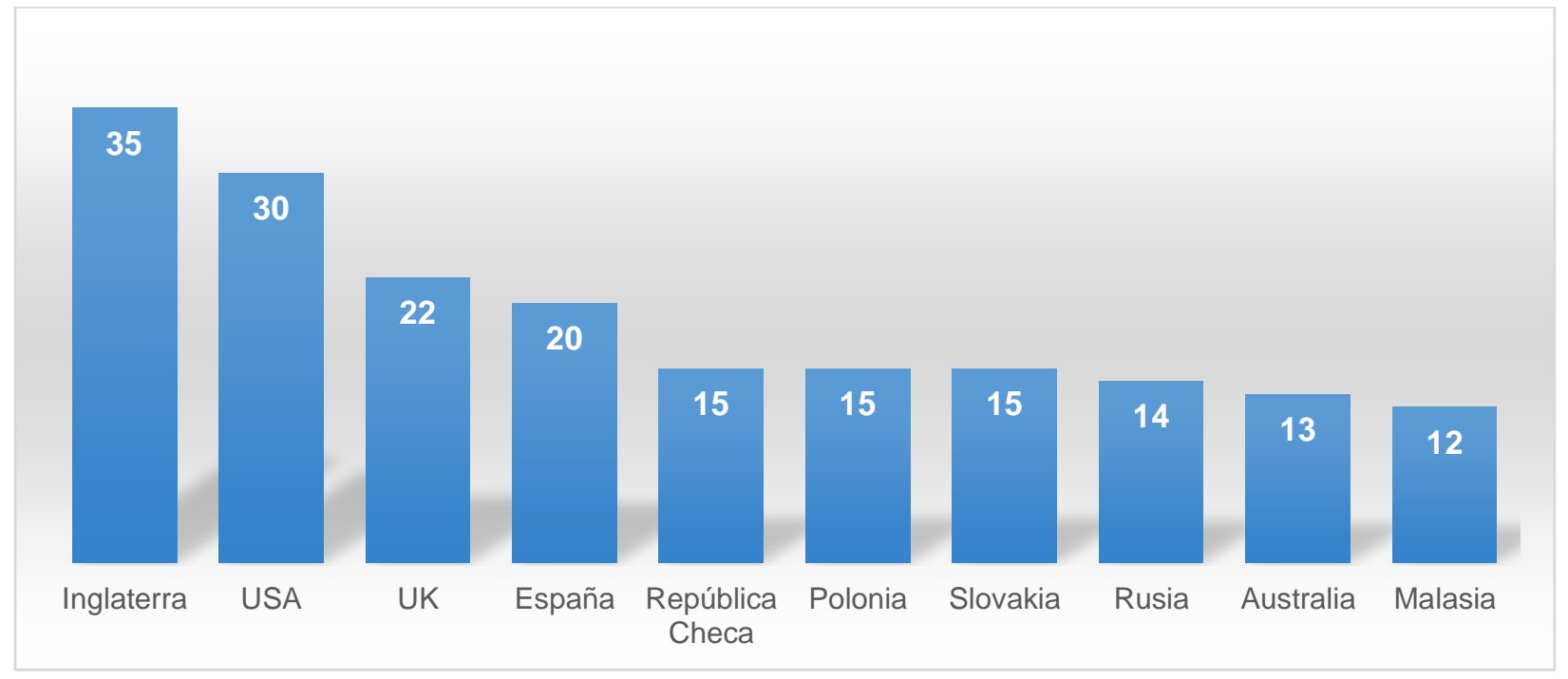


Se encontraron 82 países participantes con investigaciones de las MiPymes y el emprendimiento. Como se evidencia en la figura 3, los países con mayores aportaciones científicas pertenecen al Continente Europea y USA, destacándose Inglaterra que es el país natal del principal autor Benik Krasquini.

\section{Tabla 2}

Publicaciones con mayor número de citas

\begin{tabular}{|c|c|c|c|c|}
\hline Título & Autores & Revista & Año & Citas \\
\hline $\begin{array}{l}\text { SME } \\
\text { research: past, present and } \\
\text { future. }\end{array}$ & $\begin{array}{l}\text { Ruzzier, M.; Hisrich, } \\
\text { R.; Antoncic, B. }\end{array}$ & $\begin{array}{lr}\text { Journal of } & \text { Small } \\
\text { Business } & \text { and } \\
\text { Enterprise } & \\
\text { development } & \end{array}$ & 2006 & 293 \\
\hline $\begin{array}{l}\text { The role of SME } \\
\text { entrepreneurs innovatiness } \\
\text { and personality in the adoption } \\
\text { of innovations. }\end{array}$ & $\begin{array}{l}\text { Marcati, A.; Guido, } \\
\text { G.; Peluso, A. }\end{array}$ & Research Policy & 2008 & 149 \\
\hline $\begin{array}{l}\text { Antecedents, moderators and } \\
\text { outcomes of innovation } \\
\text { climate and open innovation: } \\
\text { An empirical study in SME'S }\end{array}$ & $\begin{array}{l}\text { Popa, S.; Soto- } \\
\text { Acosta, P.; Martínez- } \\
\text { Conesa, J. }\end{array}$ & $\begin{array}{l}\text { Technological } \\
\text { Forecasting and } \\
\text { Social Change }\end{array}$ & 2017 & 120 \\
\hline $\begin{array}{l}\text { Entrepreneurship, process } \\
\text { innovation and value creations } \\
\text { by a non-profit SME }\end{array}$ & Huarng, K.; Yu, T. & $\begin{array}{l}\text { Management } \\
\text { decision }\end{array}$ & 2011 & 85 \\
\hline $\begin{array}{l}\text { The Impact of Brand } \\
\text { authenticity on Brand trust and } \\
\text { SME growth: A CEO } \\
\text { perspective }\end{array}$ & $\begin{array}{l}\text { Eggers, F.; O’Dwyer, } \\
\text { M.; Kraus, S. }\end{array}$ & $\begin{array}{l}\text { Journal of world } \\
\text { business }\end{array}$ & 2013 & 78 \\
\hline
\end{tabular}

De la tabla 2 se observa los artículos con el mayor número de citas en donde evidencia que las temáticas de mayor interés relacionan la internacionalización y la innovación con las MiPymes. Estas investigaciones destacan que la creación de MiPymes surge en entornos cambiantes y dinámicos ante la falta de oportunidades laborales o deseo de independencia del emprendedor.

\section{Evolución del Campo Temático}




\section{Figura 5}

Período Uno 2001-2005

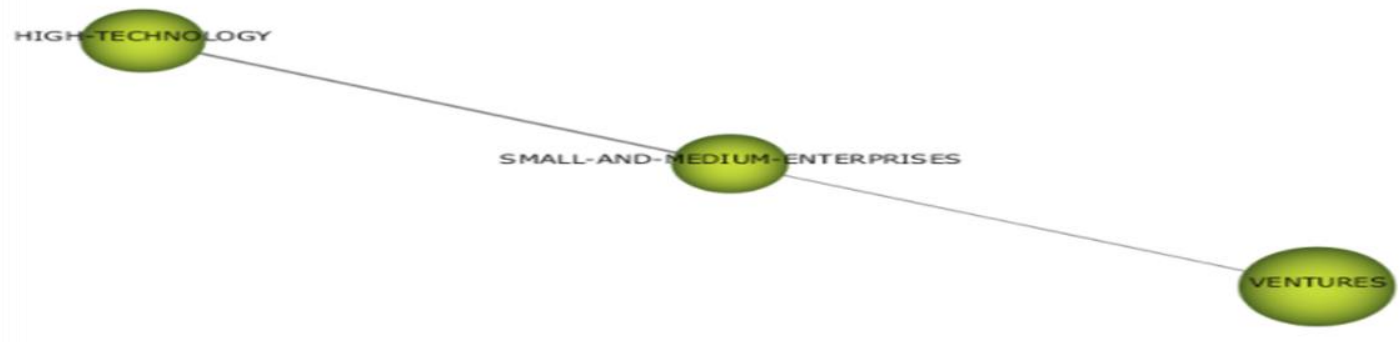

En este periodo los estudios académicos se centraron en la relación de las pymes y la era de la digitalización en un escenario tan incierto como la entrada de un nuevo milenio y sus dificultades para detectar oportunidades tecnológicas (Lee, Kim, Song, Park, \& Shin, 2014). Aunque las investigaciones de esta temática comenzaron a tomar interés desde el año 2005 aprovechando el desarrollo de la tecnología en la mejora de los procesos, la comunicación y el almacenaje de gran cantidad de información en el cloud o la nube como medida de seguridad ante softwares maliciosos.

\section{Figura 6}

Período Dos 2006-2010

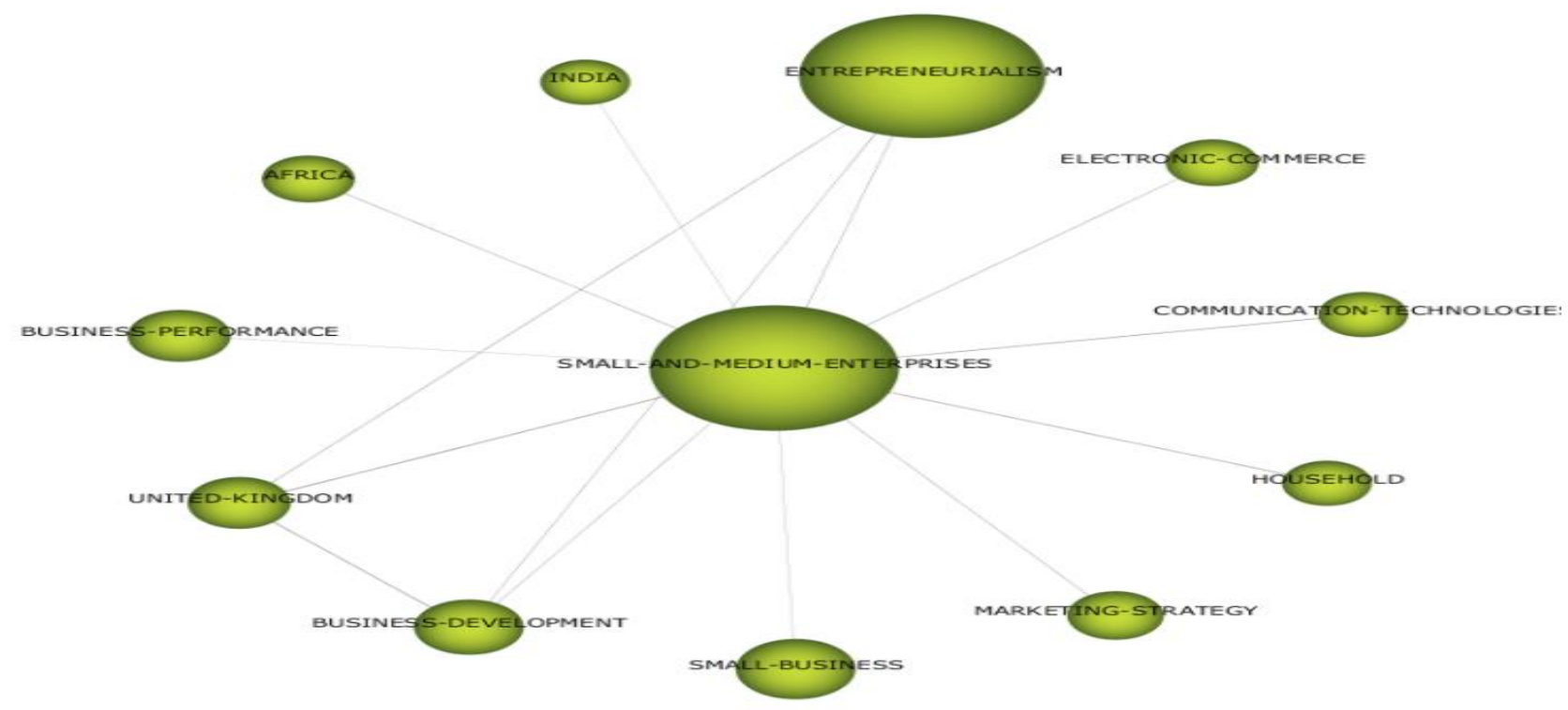


En este segundo periodo se desarrollaron 112 documentos que relacionan el uso de las tecnologías de información (tics) con las MiPymes considerándoles un elemento vital para mejorar la competitividad a través del comercio electrónico y el desarrollo de nuevos negocios. Aunque las mismas no cuenten con los recursos suficientes para la inversión en estas tecnologías. El ecomerce comienza a tener relevancia dentro de estos emprendimientos por la facilidad y rapidez para el acceso de sus productos a través del internet haciendo visibles para consumidores locales y extranjeros contando con la ayuda de las estrategias de marketing.

Lo más destacado en este periodo son los estudios realizados de comparación de países y el rendimiento empresarial de las MiPymes, siendo las investigaciones más relevantes las de Bernardino et al. (2010), Ubreziova et al. (2018) y Holz (2009).

\section{Figura 7}

Período Tres 2011-2015

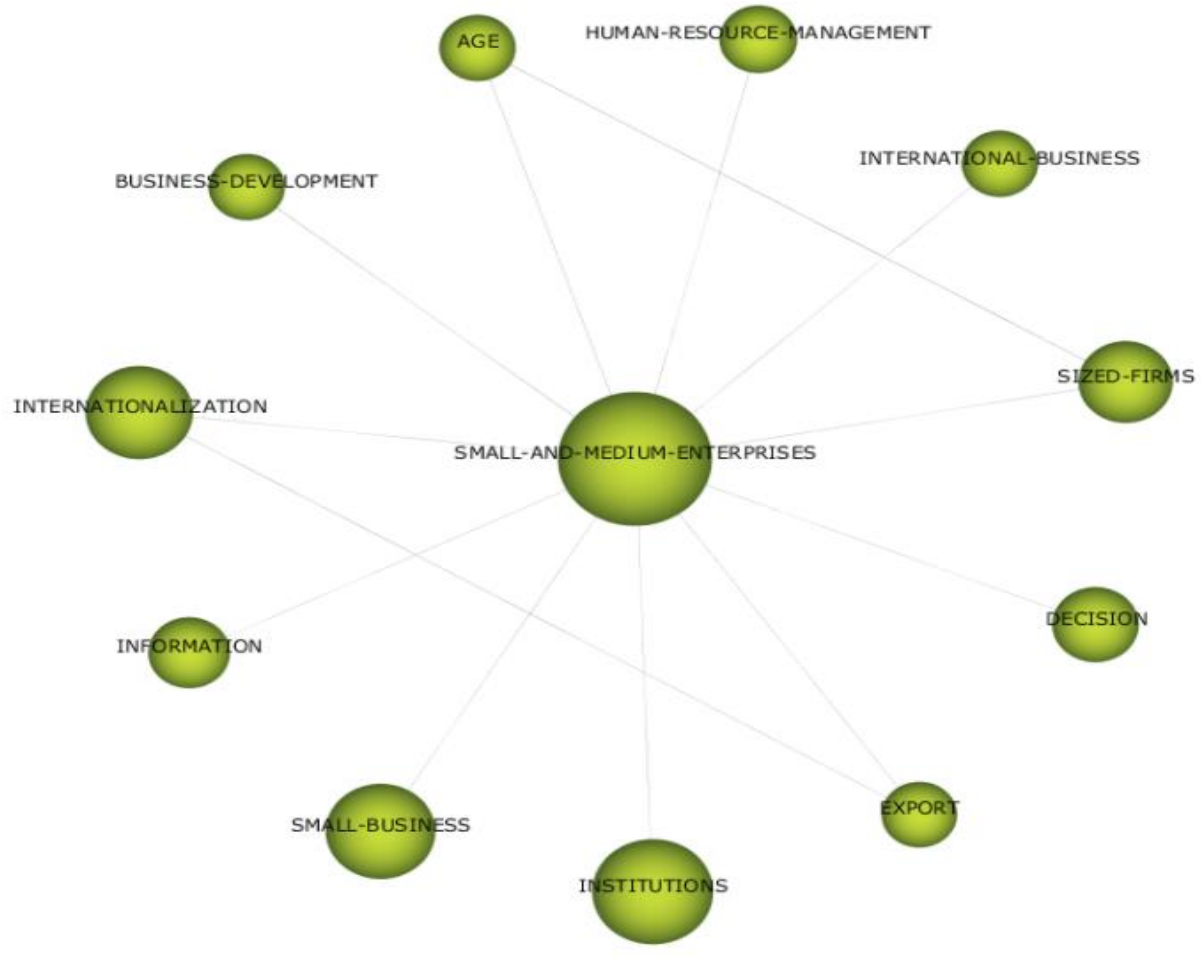

En este periodo se evidencia 350 documentos que relacionan el crecimiento de las MiPymes a través de la internacionalización como un reflejo positivo en su productividad, innovación, mejora salarial y calidad de empleo. Esta internacionalización resulta ser apoyada por redes gubernamentales en el desarrollo, creación y mejoramiento de productos o servicios no tradicionales. El articulo más citado de este período corresponde a "Entrepreneurial orientation 
and the business performance of SMEs: a quantitative study from the Netherlands" cuyos autores son Kraus et al. (2012) en donde se menciona la importancia de la Orientación Empresarial sobre el desempeño de las empresas en épocas turbulentas.

\section{Figura 8}

Período 4 2016-2020

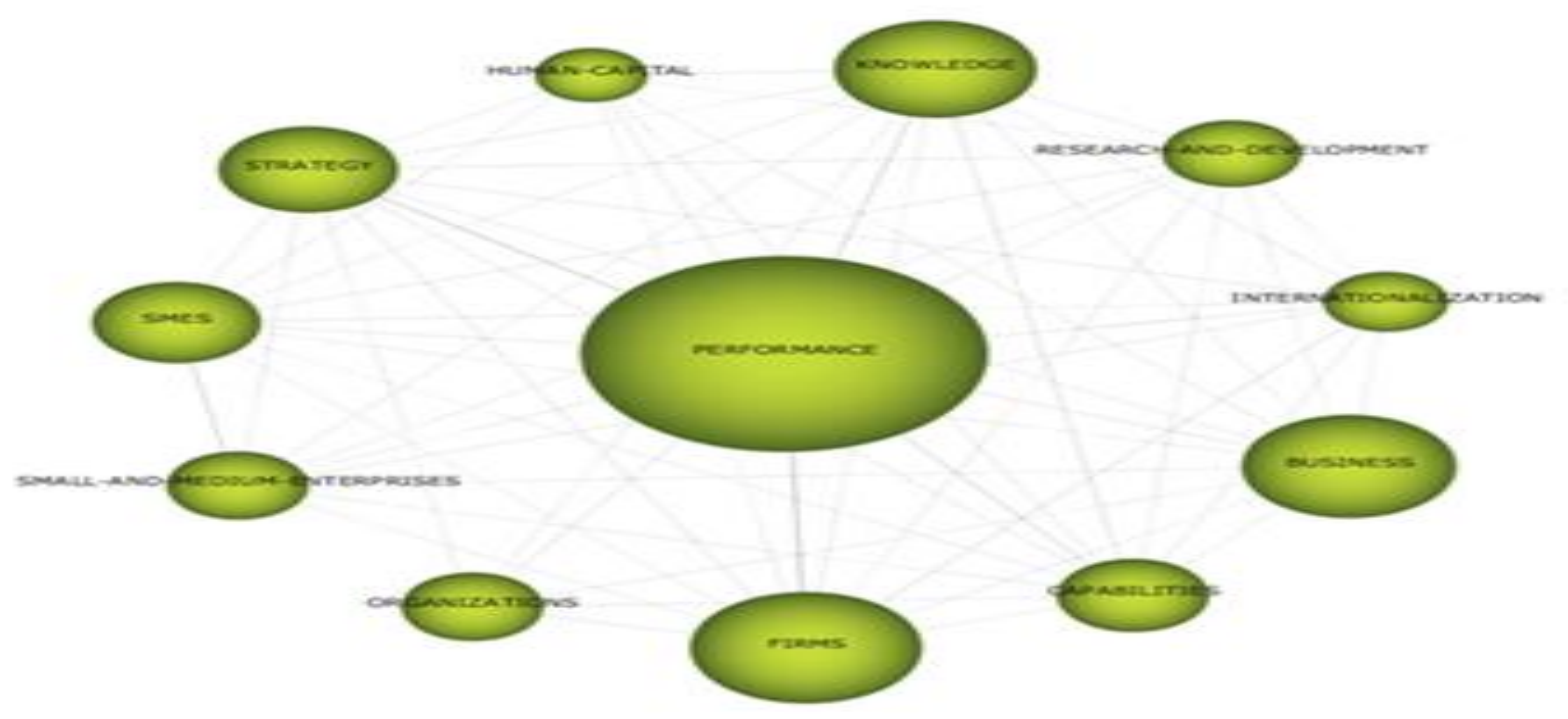

En este último período de estudio se cuenta con 425 documentos en el que enfoca sus investigaciones en la innovación y el rendimiento de las MiPymes considerando que una posible implantación de las capacidades dinámicas como herramienta estratégica mejora la toma de decisiones. Además, surgen las llamadas Born Global que son pequeñas y medianas empresas que desde su formación son internacionalizadas aprovechando la tecnología, investigación y desarrollo y el conocimiento técnico de su capital humano.

\section{Discusiones}

Las MiPymes deben tener la capacidad de generar innovación y conocimiento tácito como ventaja competitiva en entornos hostiles que le permitan desarrollarse y crecer dentro de las industrias con cuotas de mercados ya establecidas entre las líderes (Pérez-Luño, Saparito, y Gopalakrishnan, 2014). En este artículo se confirma la relación positiva y estrecha de la innovación, el conocimiento y las MiPymes. Sin embargo, destacamos que el sector más relevante para este tipo de empresas es el tecnológico, el cual está en constante cambios con avances acelerados.

En los últimos cinco años de estudio, las MiPymes suelen estar relacionadas con la economía informal de países con poca cultura tributaria, alto nivel de corrupción y bajo control 
regulatorio, tomando como referencia la paga de salarios informales a los empleados. (Williams y Horodnic, 2016).

Uno de los principales determinantes de formar una MiPymes por parte de un individuo es contar con un entorno empresarial favorable. El estudio realizado por (Dobes, Kot, Kramolis, y Sopkova, 2017) evidencia que los microempresarios creen que los gobiernos son pilar fundamental dentro de este entorno empresarial destacando que los mismos no crean políticas y leyes regulatorias para fomentar el espíritu empresarial y más bien son vistos como inhibidores de la iniciativa empresarial.

Con respecto a la organización y el tamaño de las MiPymes, este estudio evidencia que esta característica les permite ser dinámicas, flexibles y adaptables a entornos cambiantes en un mundo globalizado (Castillo-Garcia, 2019; Cleri, 2007). Además, la personalidad y la innovación juega un rol fundamenta en la formación de pymes (Marcati, Guido, y Peluso, 2008). La internacionalización de las pymes es considerada como una buena estrategia competitiva debido a que los mercados nacionales suelen ser poco rentables por el entorno familiar que surge de las competencias (Child, y otros, 2017).

Por tanto, la propuesta y creación de valor que se le da a la internacionalización está relacionada con el ofrecimiento de nuevos productos o servicios, mejores canales de comunicación o distribución, nuevas relaciones con los proveedores y clientes involucrando mejoramiento de los procesos a través de la adquisición y desarrollo de las nuevas tecnologías (Clauss, 2017; Melén Hanell et al., 2019; Ojala et al., 2018; Schu et al., 20116; Tolstoy and Agndal, 2010).

\section{Conclusiones}

Alrededor de dos tercios del empleo mundial son generados por las MiPymes (O.I.T., 2019). Estas suelen considerarse motor de crecimiento económico local (Organización para la Cooperación y Desarrollo Económico, 2017), si se encuentran bien gestionadas por organismos e instituciones adecuadas (Jegede, 2012). Por ende, los gobiernos que brinden apoyo a las mipymes deben encaminarse la sostenibilidad de las mismas (Tope-Jegede, 2020).

Existen algunos estudios que relacionan las mipymes con el emprendimiento en la revisión de la literatura (Afrin et al., 2008; Sanusi, 2003; Tijani et al., 2012; Tope-Jegede, 2020), pero ninguno de estos estudios ha estudiado la evolución conceptual desarrollado, concluyendo que su temática se encuentra relacionada con cinco temáticas centrales y básicas: conocimiento, instituciones, internacionalización, tecnología y emprendedorismo.

Los individuos que deseen formar una Mipyme deben tener como característica básica e innata la innovación y la personalidad de asumir riesgos. En un mundo globalizado las empresas compiten por valores añadidos, atributos diferenciadores más que por oportunidades, debido a esto es evidente que las MiPymes surgen como alternativas a las crisis y recesiones económicas. 
En la actualidad, las MiPymes deben considerar dentro de su estrategia empresarial involucrar sus actividades a la responsabilidad sociales y entornos sustentables para obtener un crecimiento sostenible en los mercados cambiantes y dinámicos.

Este estudio presenta algunas limitaciones como la selección de una sola base de datos (WOS), excluyendo otras como Scopus, Pubmed. Asimismo, algunos temas relacionados con las Mipymes no se encuentren evidencias por la selección de las palabras claves en la búsqueda de documentos. Como futuras líneas de investigación se puede ampliar el periodo de estudio y buscar comparaciones de estudio por países.

\section{Referencias bibliográficas}

Afrin, S., Islam, N., \& Ahmed, S. (2008). A multivariable model of microcredit and rural women entrepreneurship development in Bangladesh. International Journal of Business and Management. https://doi.org/10.5539/ijbm.v3n8p169

Bernardino, L., Jones, M., \& Morgan-Thomas, A. (2010). Resoruces and Performance in Foreign Markets: The Case of High-Technology SMEs in Portugal. Resources, Efficiency and Globalization, 249-267. https://doi.org/10.1057/9780230278028_14

Bloch, R., y Granato, L. (2007). Las PYMES y el acceso al crédito. Observatorio Iberoamericano del desarrollo local y la economía social. issue 2, https://EconPapers.repec.org/RePEc:erv:oidles:y:2007:i:2:3.

Camps, D. (2007). Estudio bibliométrico general de colaboración y consumo de la información en artículos originales de la revista Universitas Médica, período 2002 a 2006. Universitas Médicas, 48(4), 358-365. https://www.redalyc.org/articulo.oa?id=231018670002

Castillo-Garcia, P. (2019). Del emprendimiento hacia las PyMES emprendedoras en relación con su gestión financiera. En Torres-Granadillo, F. (Ed). POSSUNT: Emprendimiento Global (pp. 115-123). High Rate Gonsulting.

Child, J., Hsich, L., Elbanna, S., Karmowska, J., Marinova, S., Puthusserry, P., Tesai, T., Narooz, R. y Zhang, Y. (2017). SME International Business Models: The Role of Context and Experience. Journal of World Business. 52(4). https://doi.org/10.1016/j.jwb.2017.05.004

Clauss, T. (2017). Measuring business model innovation: conceptualization, scale development. $R \& D$ Management, 385-403. https://doi.org/10.1111/radm.12186

Cleri, C. (2007). El libro de las pymes. Granica. Argentina.

Cobo, M., López-Herrera, A., Herrera-Viedma, E., \& Herrera, F. (2012). SciMAT: A new science mapping analysis software tool. Journal of the American Society for Information Science and Technology. https://doi.org/10.1002/asi.22688

Dobes, K., Kot, S., Kramolis, J., \& Sopkova, G. (2017). The Perception of Governmental Support in The Context of Competitiveness of SMEs in the Czech Republic. Journal of Competitiveness. 9(3), 34-50. https://doi.org/10.7441/joc.2017.03.03

EKOS. (2019). Mipymes representan el $99 \%$ de negocios en Ecuador. EKOS. https://bit.ly/3vY3ffU 
García, G., y Villafuerte, F. (2015). Las restricciones al financiamiento de las pymes de Ecuador y su incidencia en la política de inversiones. Actualidad Contable Faces, 18(30),49-73. https://www.redalyc.org/articulo.oa?id=25739666004

Gómez, A., García, D., y Marín, S. (2009). Restricciones a la financiación de la pyme en México: una aproximación empírica. 57(XXIV). Universidad Autónoma Metropolitana. Azcapotzalco. México, pp. 217-38.

Guaipatín, C. (2003). Observatorio MIPYME compilación estadística para 12 países de la región. Banco Interamericano de Desarrollo, Washington. https://bit.ly/3mrfUoE

Harvey, M., \& Wendel, C. (2006). SME Scoring: Key Inititatives, Opportunities and Issues. Boletín Wold Bank, No- 38995.

Holzl, W. (2009). Is the R\&D behaviour of fast-growing SMEs different? Evidence froma CIS III data for 16 countries. Small Business Economics, 59-75. https://doi.org/10.1007/s11187009-9182-x

INEN, S. E. (2020). Mipymes y Organizaciones de Economía Popular y Solidaria son una pieza clave para la economía del país. https://www.normalizacion.gob.ec/mipymes-yorganizaciones-de-economia-popular-y-solidaria-son-una-pieza-clave-para-la-economiadel-pais/

Jegede, C. (2012). Investigation of Government's Support for Small and Medium Enterprises in Nigeria (1960-2012). En Hand book on General Studies for Higher Institutions in Nigeria (págs. 297-320). Ondo: Wesley University of Science and Technology.

Lee, Y., Kim, S., Song, I., Park, Y., \& Shin, J. (2014). Technology opportunity identification customized to the technological capability of SMEs through two-stage patent analysis. Scientometrics, 227-244. https://doi.org/10.1007/s11192-013-1216-0

Marcati, A., Guido, G., \& Peluso, A. (2008). The role of SME entrepreneurs' innovativeness and personality in the adoption of innovations. Research Policy. https://doi.org/10.1016/j.respol.2008.06.004

Melén Hanell, S., Rovira, E., Tolstoy, D., \& Özbek, N. (2019). It's a new game out there: ecommerce in internationalising retails SMEs. International Marketing Review. https://doi.org/10.1108/IMR-03-2018-0107

Granda, M. (2012). Factores determinantes de acceso y racionamiento del crédito en las MIPYMES ecuatorianas. [Tesis de Maestría, Flacso- Ecuador]. http://hdl.handle.net/10469/9246

OIT (2019). El poder de lo pequeño: hay que activar el potencial de las pymes. Organización Internacional del Trabajo, Infostories. https://www.ilo.org/infostories/esES/Stories/Employment/SMEs\#intro

OECD, O. f. (2017). Enhancing the contributions of SMEs in a global and digitalized economy OECD. OECD, Paris. https://www.oecd.org/industry/C-MIN-2017-8-EN.pdf

Ojala, A., Evers, N., \& Rialp, A. (2018). Extending the international new venture phenomenon to digital platform providers: A longitudinal case study. Journal of World Business, 725-739. https://doi.org/10.1016/j.jwb.2018.05.001

Pérez-Luño, A., Saparito, P., \& Gopalakrishnan, S. (2014). Small and Medium-Sized Enterprise's Entrepreneurial versus Market Orientation and the Creation of Tacit Knowledge. Journal of Small Business Management. https://doi.org/10.1111/jsbm.12144 
Sanusi, J. (2003). Overview of development programmes for small and medium enterprises in Nigeria. Being a paper delivered at the seminar on SMIEIS. Lagos: CBN training Centre.

Schu, M., Morschett, B., \& Swoboda, B. (2016). Internationalization speed of online retailers: A resource-based perspective on the influence factors. Management International Review, 733-757. https://doi.org/10.1007/978-3-658-18215-1_4

Tijani, A., Oyeniyi, G., \& Ogunyomi, O. (2012). The Impact of technical entrepreneurial skills on Employment Generation in Small and Medium scale enterprises in Lagos STate, Nigeria: A comparative analysis. European Journal of Humanities and Social Sciences.

Tolstoy, D., \& Agndal, H. (2010). Network resource combinations in the international venturing of small biotech firms. Technovation, 24-36.

Tope-Jegede, C. (2020). Agencies for Entrepreneurship Development and SME's Promotion in Nigeria. Agencias para el desarrollo del emprendimiento empresarial y la promoción de las pymes in Nigeria. https://doi.org/10.33110/cimexus150208

Ubreziova, I., Wach, K., \& Horvathova, J. (2008). Entrepreneurship in small and medium-sized enterprises: Comparative study between Slovakia and Poland for the years 2001-2007. Agricultural Economics-Zemedelska Ekonomika, 358-366. https://mpra.ub.unimuenchen.de/id/eprint/31507

Williams, C., \& Horodnic, I. (2016). Cross-Country variation in the participation of Small Businesses in the informal economy. Journal of Small Business and Enterprise Development. 23(1), 3-24. https://doi.org/10.1108/JSBED-02-2015-0021 